\title{
ORIGIN OF THE COSMIC X-RAY BACKGROUND
}

\author{
(Invited Discourse)
}

\section{G. SETTI}

Istituto di Fisica 'A. Righi', Osservatorio Astronomico Universitario, University of Bologna, Italy

and

\author{
M. J. REES
}

Institute of Theoretical Astronomy, University of Cambridge, England

\begin{abstract}
In this paper we review the theories which have been proposed to account for the extragalactic X-ray background. Although there is still no detailed theory, one may devise reasonable models which account in a natural way both for the intensity and the spectral shape over the whole energy band, provided that cosmological evolutionary effects are included. A model based on Compton scattering of cosmic black body photons by relativistic electrons in radio sources at large redshifts $(z \gtrsim 4)$ seems to give the most satisfactory explanation. However, the data are not yet good enough to discriminate against alternative models.

A discussion of the recent observations in the soft $\mathrm{X}$-ray region $(<1 \mathrm{keV})$, and their relevance to the physics of interstellar and intergalactic gas, is given. The available data are somewhat confusing, but it seems that this part of the spectrum may still be consistent with a simple extrapolation of the non-thermal spectrum at higher energies, though various workers have claimed the detection of a new component probably due to hot intergalactic gas. If this interpretation is correct one may deduce interesting conclusions about the state of ionization and composition of the intergalactic gas, because of the importance of the absorption effects in this energy band.

Also it appears that the Galaxy is more transparent than one would deduce from $21-\mathrm{cm}$ observations. However, due to the lack of observational data, no firm conclusions can be reached.
\end{abstract}

\section{Introduction}

In this review of theoretical interpretations of the X-ray background we shall concentrate on the astrophysical aspects of the problem. The relevant emission mechanisms and some aspects of the interpretation have already been extensively discussed in a number of reviews (Hayakawa and Matsuoka, 1964; Ginzburg and Syrovatsky, 1964a; Gould and Burbidge, 1965; Gould, 1967; Weymann, 1968; Sciama, 1968). The observations are fully reviewed by Oda (paper in this volume). The observed isotropy of the X-ray background indicates that it is predominantly extragalactic in origin (Seward et al., 1967). Some enhancement is, however, observed towards the galactic plane (Cooke et al., 1969), and several proposals have been made which could account for this contribution. We shall here consider primarily the isotropic component, which has been tentatively fitted by two power law spectra of different slopes (Gorenstein et al., 1969):

$$
\frac{\mathrm{d} N}{\mathrm{~d} E}=12.4 E_{\mathrm{keV}}^{-1.7 \pm 0.2} \text { photons }\left(\mathrm{cm}^{2} \text { ster sec } \mathrm{keV}\right)^{-1}
$$


from 1 to $40 \mathrm{keV}$, and approximately

$$
\frac{\mathrm{d} N}{\mathrm{~d} E} \simeq 20 E_{\mathrm{keV}}^{-2} \text { photons }\left(\mathrm{cm}^{2} \text { ster sec } \mathrm{keV}\right)^{-1}
$$

above $60 \mathrm{keV}$.

There is a substantial spread in the fluxes reported by various experimental groups, so that these precise spectra should not be taken too literally, but it definitely seems that the slope changes around 20-40 keV. Bleeker and Deerenberg (paper in this volume), who have a homogeneous set of observations between 20 and $220 \mathrm{keV}$, obtain a straight spectrum with the much steeper slope $2.45 \pm 0.1$ over this range, and conclude that any spectral break must occur at $\$ 20 \mathrm{keV}$.* In view of all the uncertainties, it is difficult to specify either the position of the break, or the slope of the spectrum in various energy ranges, with any precision. As we shall see, detailed knowledge of the spectrum would be important in deciding between various models.

A background X-ray flux has also been reported below $1 \mathrm{keV}$, but at the moment it is impossible to give any reliable figures either for the intensity or the behaviour of the spectrum (Oda, paper in this volume). In view of the astrophysical and cosmological interest which attaches to these soft X-ray observations, we devote a special section of our review to this topic.

In the following sections we attempt to present a critical discussion of current ideas on these topics.

\section{Energy Requirements for Universal X-Ray Background}

The energy density of the isotropic background from 2 to $1000 \mathrm{keV}$, with spectrum (1), is

$$
\sim 6 \times 10^{-5} \mathrm{eV} \mathrm{cm}^{-3}
$$

Neglecting cosmological effects, this figure represents the mean energy input per unit volume required to generate the flux. If the bulk of this radiation were emitted at redshifts $z \simeq \bar{z}$, the energy input per unit coordinate volume would be increased by $(1+\bar{z})$ (see Section 4).

It is interesting to compare this with the energy densities of background radiation in other energy bands. The estimated metagalactic energy density at radio wavelengths $(1-1000 \mathrm{MHz})$ is $\sim 10^{-7} \mathrm{eV} \mathrm{cm}^{-3}$; the energy density of $2.7 \mathrm{~K}$ black-body radiation is $\sim 0.3 \mathrm{eV} \mathrm{cm}^{-3}$; and the metagalactic background at optical wavelengths is $\sim 10^{-2} \mathrm{eV} \mathrm{cm}^{-3}$ (the infrared background is highly uncertain, but is unlikely to exceed that at optical wavelengths (Low and Tucker, 1968)). It is thus clear that the energy going into X-rays is small compared with the output of typical galaxies at longer wavelengths. The problem raised by the X-ray background is therefore not primarily an energetic one - the problem is rather to understand how $\sim 0.5 \%$ of the

* Brini et al. (paper in this volume), who obtain a much less steep spectrum more in agreement with $\left(1^{\prime \prime}\right)$, also do not find any appreciable break down to $25 \mathrm{keV}$. 
radiation from sources* can be channelled into hard photons with the observed spectrum.

\section{Models not Involving Cosmological Evolution}

It is convenient to divide the proposed models into two categories:

(a) those which interpret the X-ray background as the integrated effect of discrete sources;

(b) those which invoke emission mechanisms that operate throughout intergalactic space, or at least in very extended diffuse regions.

In our discussion of (a), we shall merely consider the likely contribution from various classes of objects. (The emission mechanisms for discrete sources are reviewed by Felten, paper in this volume.) We shall consider (b) at greater length, because it is in this case possible to attempt to fit the observed spectrum. We adopt this same procedure in Section 4, where evolutionary models are discussed.

\section{A. DISCRETE SOURCE MODELS}

The X-ray emission from sources in the Galaxy is $10^{39}-10^{40} \mathrm{ergs} \mathrm{sec}^{-1}$ (Oda, 1965; Silk, 1969). In order to estimate the likely contribution from all normal galaxies, it is convenient to express this output as $5 \times 10^{-6}-5 \times 10^{-5} \mathrm{ergs}_{\mathrm{gm}}^{-1} \mathrm{sec}^{-1}$. Assuming all galaxies to have the same mean X-ray emission per unit mass, and that the smoothed-out density of galaxies has the value $\sim 3 \times 10^{-31}$ estimated by Oort (1958), we obtain an X-ray energy density of $3 \times 10^{7}-3 \times 10^{-6} \mathrm{eV} \mathrm{cm}^{-3}$. Even taking the optimistic estimate of $\sim 10^{40}$ ergs $\sec ^{-1}$ for the output of our Galaxy, we still fall short of the observed X-ray background intensity by a factor $\sim 20$. Unless other galaxies typically emit X-rays more strongly than our own, or unless the density of faint galaxies greatly exceeds Oort's estimate, it seems unlikely that the observed background could be produced. However, in view of our ignorance of the X-ray properties of other galaxies, we cannot exclude this possibility completely. One would have to invoke galactic sources with power-law spectra (e.g. the Crab) rather than sources like Sco X-1 which are better fitted by exponential spectra. (The isotropy of the $\mathrm{X}$-ray background is also evidence against the idea that it arises from normal galaxies - at visible wavelengths the background anisotropy is very large because starlight from our Galaxy swamps the extragalactic contribution.)

The X-rays may of course come from a rarer class of sources more powerful than ordinary galaxies - e.g. quasars or radio galaxies. No quasars have yet been definitely detected as X-ray sources, but the flux from 3C 273 could be $\sim 7 \times 10^{45} \mathrm{ergs} \mathrm{sec}^{-1}$ in the 1-10 $\AA$ band (Friedman and Byram, 1967). If all quasars in fact emitted a flux of this strength, the required space density would be $\sim 10^{-6} \mathrm{Mpc}^{-3}$. On the other hand, Schmidt (1968) estimates the actual local space density of objects like 3C 273 to be only $\sim 10^{-9} \mathrm{Mpc}^{-3}$, so that in this case also there appears to be a large discrepancy. Alternatively one may consider radio galaxies as candidates. The local

\footnotetext{
* Since any radiation present in the early dense phases of an evolving universe would have been
} thermalized, the X-ray background clearly cannot be primeval. 
space density of radio galaxies emitting $\gtrsim 10^{42} \mathrm{ergs} \mathrm{sec}^{-1}$ at radio wavelengths is a few times $10^{-6} \mathrm{Mpc}^{-3}$ (Schmidt, 1966). The mean X-ray flux from these would need to be $\sim 1000$ times greater than their radio output. Present observations show that this cannot be true for the nearby radio galaxies Centaurus A (Haymes et al., 1969) and Virgo A (Friedman and Byram, 1967). We would therefore have to appeal exclusively to the strongest radio sources $\left(\gtrsim 10^{44} \mathrm{ergs} \mathrm{sec}^{-1}\right.$ in the radio band), which, according to Schmidt (1966) have a local space density of $10^{-8}-10^{-9} \mathrm{Mpc}^{-3}$.

It thus appears difficult to account for the X-ray background in terms of known types of discrete sources, without appealing to cosmological evolutionary effects.

\section{B. DIFFUSE PROCESSES}

\section{Compton Radiation}

The bulk of the radiation energy in intergalactic space resides in the microwave region, and has been interpreted as having the spectrum of a $2.7 \mathrm{~K}$ black body. We confine attention to Compton scattering of these microwave photons. (Scattering of optical photons is relatively unimportant (Felten and Morrison, 1966).)

When isotropic radiation is scattered by electrons of Lorenz factor $\gamma$, the energy of the photons is raised by a factor $\sim \gamma^{2}$. The black-body photons have typical energies $3 k T \simeq 10^{-3} \mathrm{eV}$. In order to obtain $\mathrm{X}$-rays in the range $1 \mathrm{keV}-1 \mathrm{MeV}$, electrons are needed with $\gamma$ in the range $10^{3}-10^{4.5}$. These same electrons would emit radio frequency synchrotron radiation in magnetic fields with the strength $10^{-4}-10^{-6} \mathrm{G}$, which are believed typical of radio galaxies. The X-ray and radio spectra would have similar shapes, a power-law electron spectrum $\mathrm{N}(E) \mathrm{d} E \propto E^{-\beta} \mathrm{d} E$ giving rise to radiation spectra with slope $-(\beta-1) / 2$ (or photon number spectra with slope $-((\beta+1) / 2)$. This immediately suggests the possibility of relating $\mathrm{X}$-ray and radio observations.

The radio background emission in the direction of minimum sky brightness is $\sim 80 \mathrm{~K}$ at $178 \mathrm{MHz}$ (Turtle and Baldwin, 1962). This gives a definite upper limit to the extragalactic radio background. It is usually assumed, in fact, that the true extragalactic contribution is no more than 20 or $30 \mathrm{~K}$, the remainder being due to the galactic halo and disc (Bridle, 1967a). About half of this extragalactic component is contributed by sources already seen in the source counts (Pooley and Ryle, 1968). Therefore, if the electrons producing the X-ray background via Compton scattering are not simultaneously to produce a radio background exceeding $\sim 10 \mathrm{~K}$ at $178 \mathrm{MHz}-$ and this is a conservative supposition - we may evaluate an upper limit to the magnetic field by comparing the synchrotron and Compton fluxes at two frequencies $v_{R}$ and $v_{X}:$

$$
\frac{F\left(v_{X}\right)}{F\left(v_{R}\right)}=Q\left(\beta, w_{\mathrm{ph}}\right)\langle H\rangle^{-\frac{\beta+1}{2}}\left(\frac{v_{X}}{v_{R}}\right)^{-\frac{\beta-1}{2}},
$$

where $\beta$ is the exponent in the differential electron spectrum, $\langle H\rangle$ the average magnetic field, $w_{\mathrm{ph}}$ the energy density in the black-body radiation, and $Q$ is a function 
of $\beta$ and $w_{\mathrm{ph}}$ (Bergamini et al., 1967). For $\beta \approx 3$ it turns out that

$$
\langle H\rangle \lesssim 1.7 \times 10^{-7} \mathrm{G} \text {. }
$$

More generally, this sets a limit to the mean-square field seen by an electron over its lifetime. This is far below the estimated magnetic field in our Galaxy, or in any discrete sources, so we conclude that the number of electron actually within these sources is insufficient to produce the X-ray background by Compton scattering of 2.7 K microwave photons. E.g., if the field were $\sim 10^{-6} \mathrm{G}$, the number of electrons required to produce the whole radio background would give an associated X-ray background only $\sim 1 \%$ of what is required. (For this reason, X-ray emission from the galactic halo would be negligible.)

Felten and Morrison (1966) were the first to propose that there may be an intergalactic flux of relativistic electrons sufficient to generate the X-ray background. The time $t_{\text {comp }}$ in which an electron loses half its energy by scattering black-body photons is

$$
t_{\text {comp }} \approx 10^{12} / \gamma \text { years }
$$

In intergalactic space, Compton losses would dominate all others, so the relevant electrons have lifetimes $10^{9}-3 \times 10^{7}$ years. The fact that this is short compared with the Hubble time means that almost all the energy produced in the form of relativistic electrons with $\gamma \gtrsim 10^{3}$ could have been converted into X-rays.

Equation (4) requires that, if radio sources with magnetic fields of, say, $3 \times 10^{-6} \mathrm{G}$, dimensions $\sim 50 \mathrm{kpc}$, and radio power $\sim 10^{42} \mathrm{erg} \mathrm{sec}^{-1}$, generate enough electrons to give the X-ray background, the electrons must escape from the radio volume in less than $10^{6}$ years. Taking Schmidt's (1966) estimate of $\sim 10^{9}$ years for the lifetime of such radio sources, the energy requirements amount to $\sim 10^{61}$ ergs in relativistic electrons alone. Even if the energy of the associated protons were $\sim 100$ times greater, this could not be definitely excluded on energetic grounds, provided that the acceleration mechanism is highly efficient (e.g., the collapse of $\sim 10^{10} M_{\odot}$ would yield $10^{63} \mathrm{ergs}$ for $\sim 5 \%$ efficiency).

Such models can be further tested by comparing the spectra of radio sources and the X-ray background. The average radio spectral index of radio galaxies is $\alpha=0.8$. (The corresponding differential electron spectrum has $\beta \approx 2.6$.) If electrons escaped into intergalactic space with this spectrum and were thereafter subject only to Compton losses, the X-ray background would have a steeper slope $\alpha=1.3$ right down to $\sim 10 \mathrm{eV}$ (Felten and Morrison, 1966), since the lifetimes of the electrons which radiate at higher energies are shorter than the Hubble time (from (5)). Therefore, if the X-ray background is indeed flatter below $\sim 20 \mathrm{keV}$, further consideration of the escape mechanism, or of the low energy spectrum within the source, is required in order to reconcile this model with the observations.

Brecher and Morrison (1969) have considered whether normal galaxies, rather than radio galaxies, could generate the required relativistic electrons. The energy which must be injected amounts to

$$
\sim 3 \times 10^{-22} \mathrm{eV} \mathrm{cm}^{-3} \mathrm{sec}^{-1} \text {. }
$$


In estimating the contribution from an individual galaxy one needs to know the space density of galaxies involved. These authors assumed the radio luminosity function proposed by Sholomitskii (1968):

$$
n(P) \mathrm{d} P=1.3 \times 10^{23} P^{-2.18} \mathrm{~d} P^{*},
$$

where $P\left(\mathrm{~W}(\mathrm{~Hz} \text { ster })^{-1}\right)$ is the radio power at $178 \mathrm{MHz}$, and $n(P)$ the source number density in $\mathrm{Mpc}^{-3}$. This luminosity function is not well established in the range $P \simeq 10^{20}-10^{22} \mathrm{~W}(\mathrm{~Hz} \text { ster })^{-1}$ spanned by normal galaxies. However, even granting its validity, Brecher and Morrison's work seems to entail several difficulties.

If one cuts off the luminosity function(7) below a value $P_{1}$ such that

$$
\int_{P_{1}}^{\infty} n(P) \mathrm{d} P \simeq 0.03,
$$

the density of normal galaxies given by Allen (1963), one finds that $P_{1} \simeq 8 \times 10^{20} \mathrm{~W}$

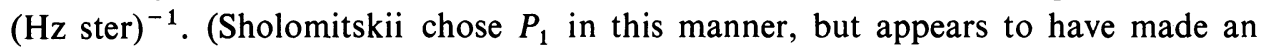
arithmetical error in evaluating it.) Brecher and Morrison, however, extrapolate (7) right down to $P=10^{20} \mathrm{~W}(\mathrm{~Hz} \text { ster })^{-1}$, which gives a source density $\sim 0.27 \mathrm{Mpc}^{-3}$. Even taking this high density of galaxies, we find that each has to produce

$$
\sim 5 \times 10^{40} \mathrm{erg} \mathrm{sec}^{-1} \text {. }
$$

This is a measure of the rate at which relativistic electrons must be produced. The observed energy density of relativistic electrons above $1 \mathrm{BeV}$ in the disc of our Galaxy is $\sim 10^{-2} \mathrm{eV} \mathrm{cm}^{-3}$. Thus, if they are generated at the rate (9), a volume of $\sim 1 \times 10^{68} \mathrm{~cm}^{3}$ could be filled or replenished in $\sim 10^{6}$ years. This volume is comparable with that of the whole galactic halo. (If the electrons are confined only in the disc, the escape time would be no more than $\sim 10^{5}$ years.) As Brecher and Morrison point out, these energy requirements are not impossible, even allowing 100 times as much energy for the replenishment of the associated protons, which presumably escape in the same timescale. We note, however, that in this model the cosmic-ray primaries could not have passed through the $3-4 \mathrm{gm} \mathrm{cm}^{-2}$ (Shapiro and Silberberg, 1968) of matter implied by the composition data above $\sim 1 \mathrm{BeV}$ per nucleon**; for the usually assumed density of $\sim 1$ atom $\mathrm{cm}^{3}$ in the disc and $\$ 10^{-2} \mathrm{~cm}^{-3}$ in the halo, this requires confinement for $\sim 10^{6}$ years or $\gtrsim 10^{8}$ years respectively.

The difficulties with this model are aggravated when we remember that most of the galaxies included in the luminosity function (7) are smaller than our own. If we follow Brecher and Morrison in deducing the electron density in these galaxies from an equipartition argument, assuming their storage volume to be similar to that of our Galaxy, we find that the average galaxy contributes only $\sim 10 \%$ of the electron flux

* The source counts in fact indicate that the luminosity function depends on redshift, the proportion of powerful sources being higher in the past (Longair, 1966). When this is allowed for, it is no longer true that $95 \%$ of the radio background would come from normal galaxies, as is stated by Brecher and Morrison.

** Unless one postulates that all this matter has been traversed within the sources. 
from our own. (If, on the other hand, we assume that the storage volumes are proportional to $P$ and the escape times similar, we lose $t w o$ orders of magnitudes.) Consequently, even if (7) can be validly extrapolated down to $P \simeq 10^{20} \mathrm{~W}(\mathrm{~Hz} \text { ster })^{-1}$, we have severe doubts about this model.

The slope of the electron spectrum in the Galaxy changes at $\sim 3 \mathrm{BeV}$. This is associated with a break in the galactic synchrotron background spectrum at a few hundred MHz. A similar bend is observed in the radio spectra of certain other normal galaxies (Lang and Terzian, 1969). It is interesting that the corresponding bend in the Compton X-ray spectrum would occur at $\sim 40 \mathrm{keV}$, where such a break indeed appears (but see Section 1). Brecher and Morrison point out that the observed $\mathrm{X}$-ray background spectrum could be explained if there were a break in the electron spectrum at $3 \mathrm{BeV}$ in all galaxies. They have to suppose that this break is characteristic of the injected spectrum, and therefore that all the sources (e.g. supernova remnants) in all galaxies inject identical spectra. The break has a natural interpretation in terms of losses if one takes the traditional estimates for the confinement time; but of course we can no longer invoke this explanation in the theory of Brecher and Morrison, where the electrons must escape more rapidly.

\section{Thermal Bremsstrahlung}

Plasma at temperatures $\gtrsim 10^{6} \mathrm{~K}$ emits thermal bremsstrahlung in the X-ray band. A significant background flux could arise from a general intergalactic medium with density $\sim 10^{-5}$ particles $\mathrm{cm}^{-3}$ or from isolated regions of higher density (e.g. gas within clusters of galaxies). As the X-ray background above $1 \mathrm{keV}$ has a typically non-thermal spectrum, we cannot attribute it to thermal bremsstrahlung without postulating either an ad hoc distribution of temperatures up to $\sim 10^{9} \mathrm{~K}$, or a very special thermal history for the intergalactic gas.

This process may, however, contribute to the X-ray background at energies $\lesssim 1 \mathrm{keV}$, and we shall discuss it further in Section 5 .

\section{Non-Thermal Bremsstrahlung}

a. Electrons. - A suprathermal electron of energy $\varepsilon$ moving through a cool plasma emits bremsstrahlung photons with a spectrum which falls off sharply above a frequency such that $h v \simeq \varepsilon$ and is approximately flat at lower energies. Non-thermal electrons with a power-law spectrum would give rise to radiation which also obeys a power-law. The radiation spectral index is the same as that of the electrons when the latter are non-relativistic, and is one power flatter when they are relativistic. So, in order to explain the X-ray background, one may postulate a non-relativistic electron flux with spectral index 0.7 below $20 \mathrm{keV}$ and $\sim 1$ above $20-40 \mathrm{keV}$ (the electron number spectrum per unit volume would be half a power steeper).

The lifetime of an electron of energy $\varepsilon_{\mathrm{keV}}$ in a gas of density $n$ is

$$
t_{L} \approx \frac{6 \times 10^{7}}{n} \varepsilon_{\mathrm{keV}}^{3 / 2}
$$


For $n=n_{0} \simeq 10^{-5} \mathrm{~cm}^{-3}$ this is less than the Hubble time for energies $\$ 1000 \mathrm{keV}$. However, it is important to note that most of the electrons energy is transferred directly to the thermal gas, and only a small proportion appears as bremsstrahlung emission. The actual fraction is directly proportional to $\varepsilon$, and is $\sim 10^{-2}$ for $\varepsilon \simeq 10 \mathrm{keV}$. This means that the energy requirements are $\sim 10^{2}$ times higher than in the Compton case. Also, the energy input into the intergalactic gas would heat it to $\gtrsim 10^{6} \mathrm{~K}$, in which case thermal X-rays would also be emitted. Also, the only way of interpreting the break in the spectrum (apart from considering evolution, as we shall in the next section) is to take a gas density $n \ll 10^{-5} \mathrm{~cm}^{-3}$, so that the Hubble time is $\sim t_{L}$ for $\varepsilon \simeq 20-40 \mathrm{keV}$. The energy requirements then amount to $>1 \mathrm{eV} \mathrm{cm}^{-3}$ in sub-relativistic electrons, which seems an exorbitant value for intergalactic space (Silk, 1969).

b. Protons. - It is also possible to obtain photons of energy $h v$ from collisions of suprathermal protons of energy $\varepsilon \gtrsim\left(m_{p} / m_{e}\right) h v$ with thermal electrons. This is the so-called 'innerbremsstrahlung' process described by Hayakawa and Matsuoka (1963). Taking $n=10^{-5} \mathrm{~cm}^{-3}$ the energy requirements are $\sim 20$ times greater than for electrons*, quite apart from the difficulty of fitting the break in the spectrum unless an intergalactic gas density substantially greater is assumed. Therefore this process seems unlikely to play much of a role as far as the isotropic background is concerned, though it may make a small contribution $(\$ 10 \%)$ to the diffuse X-ray emission from the galactic disc (Boldt and Serlemitsos, 1969).

\section{Models Incorporating Cosmological Evolution}

The preceding discussion demonstrates the difficulty of accounting for the observed intensity and spectrum of the X-ray background in terms of processes occurring at the present epoch, without making rather extreme assumptions regarding the energy sources. Also there is no obvious interpretation for the bend in the spectrum. Various workers have consequently been led to propose models which relegate the production of the X-rays to a remote epoch. In evolutionary cosmologies, the intergalactic gas would have been denser in the past, and the primeval radiation field more intense. Moreover, there is evidence from the distribution of quasars (Véron, 1966a, b; Schmidt, 1968; Rowan-Robinson, 1968) and from the radio source counts (Davidson and Davies, 1964a, b; Longair, 1966; Rowan-Robinson, 1967; and the excellent review paper by Ryle, 1968) that violent events were more frequent at earlier epochs, so that the energy problem may be less embarrassing.

All of the models mentioned in Section 3 have been extended to incorporate evolutionary effects, and we shall discuss each briefly. But first we remember, as mentioned in Section 2, that the energy requirements per unit volume are increased in proportion to the mean redshift factor $(1+\bar{z})$. The observed photons would of course have been generated with energies higher by $(1+\bar{z})$. The expansion timescale $t_{\text {exp }}$ for the universe would have been shorter in the past (except in Lemaitre-type models).

\footnotetext{
* It is true that the energy of a proton is $\sim 1800$ that of an electron, but the proton range is corre-
} spondingly greater. 
For the Einstein-de Sitter model (with deceleration parameter $q_{0}=\frac{1}{2}$ ) $t_{\exp } \propto(1+z)^{-3 / 2}$, and for a low-density model $\left(q_{0} \simeq 0\right), t_{\exp } \propto(1+z)^{-1}$. These two effects require that the power output in X-rays would need to have been much greater in the past in order for the dominant contribution to come from large redshifts (in fact the power input must depend on $z$ more steeply than $(1+z)^{2.5}$ for an Einstein-de Sitter model).

\section{A. DISCRETE SOURCE MODELS}

Silk $(1968,1969)$ has considered evolution of the X-ray power of normal galaxies with redshift. It is plainly possible to account for any observed flux by postulating the requisite amount of evolution. The only basis for the assumed evolution is an analogy with the inferred evolution of strong radio galaxies. However, the radio power of normal galaxies could not evolve in the manner hypothesised for the X-ray power without violating the observed limit on the extragalactic radio background.

There is strong evidence for a sharp increase in the coordinate density of powerful quasars, at least out to $z \simeq 2$. According to Schmidt (1968) this density is $\sim 1000$ times greater at $z \simeq 2$ than it is locally. Even if this strong evolution continued out to $z \simeq 3$, and all QSS emit X-rays at the maximum level possible for $3 \mathrm{C} 273$, one fails by a factor $\sim 10$. However, if one includes all the radio-quiet objects and assumes that they evolve equally strongly, one may perhaps fit the observed flux. It is not worthwhile to pursue this possibility further until the X-ray intensities and spectra of at least a few QSS are known. At present there is still no confirmation of X-ray emission from 3C 273, which is, intrinsically as well as apparently, one of the strongest known QSS.

In the case of ordinary radio galaxies, we cannot assume such strong evolution as for QSS and other very strong sources, because of the limit set by the radio background (Longair, 1966). Their contribution to the X-ray background would be unimportant unless their X-ray power evolved more strongly than their radio power.

\section{B. DIFFUSE PROCESSES}

\section{Compton Radiation}

In a 'big bang' cosmology, the black-body radiation temperature $T \propto(1+z)$ and the energy density

$$
w_{\text {ph }} \propto(1+z)^{4} \text {. }
$$

This means that at large redshifts the energy of relativistic electrons can be converted more rapidly into X-rays. In fact we can generalise (5) to

$$
t_{\text {comp }} \approx \frac{10^{12}}{\gamma(1+z)^{4}} \text { years. }
$$

In consequence, the permitted magnetic energy density given by (4) can be increased in proportion to $w_{\mathrm{ph}}$, so that, if the $\mathrm{X}$-rays come from redshifts $\bar{z}$, we have

$$
\langle H\rangle \lesssim 1.7 \times 10^{-7}(1+\bar{z})^{2}
$$

(we assume a spectral index $\alpha \simeq 1$ ). 
Therefore, for $\bar{z} \gtrsim 2$, values of $\langle\mathrm{H}\rangle \gtrsim 10^{-6} \mathrm{G}$ are allowed. These magnetic fields are strong enough to make the radio galaxies themselves (at large $z$ ) good candidates as sources of the X-ray background. We may then connect the X-rays to observations of the evolution and spectra of radio sources, as proposed by Bergamini et al. (1967). Similar conclusions have been reached independently by Brecher and Morrison (1967) and Rees (1967).*

The steep slope of the $\log N / \log S$ curve can be explained if a class of powerful sources were more numerous in the past. A consequence of this interpretation is that a large proportion of the observed sources which contribute to the radio background, should have large redshifts. The bulk of the emission could even come from redshifts $z \simeq 5$. At the moment it is not clear whether quasars alone, or whether radio galaxies also partake in this evolution (Ryle, 1968). However, it is certainly consistent with the present data to assume that a large proportion of the extragalactic radio background comes from extended sources (though the extent to which such sources contribute to the counts depends on their individual power). If these sources are to contribute the $X$-ray background the magnetic field must, from $\left(4^{\prime}\right)$, be $\lesssim 10^{-6} \mathrm{G}$ at $z \simeq 2$ and $\lesssim 6 \times 10^{-6} \mathrm{G}$ at $z \simeq 5$ (though higher fields would be permitted if the electrons escape into a weaker field before losing all their energy). There are no reliable estimates of the magnetic fields within any extended radio volumes, but these required fields are well below the equipartition field in the strong sources. However, there is no basis other than the assumption of minimum energy for assuming an equipartition field. Indeed, observations of sources where synchrotron self-absorption occurs show that - at least in these compact objects - the field is in fact far below the equipartition strength (Hornby and Williams, 1966; Williams, 1966; Bridle, 1967b; but see Kellermann and Pauliny-Toth, 1969). There is no problem as far as the basic energetics are concerned in assuming weaker magnetic fields in the sources. We turn now to consider the interpretation of the observed X-ray background spectrum in the Compton theories.

Observations of individual sources with $z \lesssim 1$ indicate that the injected electron spectrum has index $\beta \simeq 2.6$, corresponding to a radio spectral index $\alpha \simeq 0.8$ (Kellermann, 1966). If we suppose tentatively that this is also the injection spectrum in the sources at larger $z$, then the integrated radio and X-ray spectra would both be steepened by Compton losses, and we would expect the $\alpha$ to be closer to 1.3 , at least at the higher energies where the Compton losses would dominate.

To account for the position of the break, one needs a more specific source model allowing for the competing adiabatic losses (which are relatively more important at low energies). Such a model was proposed by Rees and Setti (1968), and we summarise it briefly below.

The model is based on the hypothesis that the evolution of extended radio sources is controlled by two main factors: (i) dynamical interaction with the intergalactic medium, and (ii) Compton losses. The development of a source from the initial explosion is divided into two phases: Phase $\mathrm{I}$, a free relativistic expansion to a radius

* It was not possible to make this connection in the previous discussion of radio galaxies, since we did not then specify the emission mechanism, and could not predict its dependence on $z$. 
$R_{1} \simeq 10^{4} \mathrm{pc}$, at which stage the internal field is assumed to be $H_{1} \simeq 10^{-5} \mathrm{G}$; and Phase II, a slower (but still rapid in cosmic time) continuing expansion against the 'ram pressure' of the intergalactic gas. For a model with internal energy $\sim 10^{60}$ ergs at the beginning of Phase II which is expanding into an external gas of density $2 \times 10^{-29}(1+z)^{3} \mathrm{gm} \mathrm{cm}^{-3}$, the timescale for the expansion from $R_{1}$ to, say, $2 R_{1}$ is

$$
t\left(2 R_{1}\right) \simeq 1 \times 10^{5}(1+z)^{3 / 2} \text { years } .
$$

During this time the fast particles must give up much of their energy in the adiabatic expansion (this energy may eventually heat the intergalactic gas). A break in the spectrum at $20 \mathrm{keV}$ could be explained if the sources making the main contribution to the background are at a redshift such that $t_{\text {comp }}$ (given by $\left(5^{\prime}\right)$ ) for the relevant electrons (which have $\left.\gamma \simeq 4 \times 10^{3}\right)$, ${ }^{*}$ is equal to $t\left(2 R_{1}\right)$ (Felten and Rees, 1969). The redshift derived in this way is $\sim 4$. . $^{* *}$ The parameters of the model - the intergalactic density, the internal energy, and the initial magnetic field - are very uncertain, so it would be unjustifiable to attach too much significance to this particular redshift. Nevertheless it is gratifying that a plausible choice for these parameters can explain not only the intensity, but also the spectrum, of the X-ray background.

It is worth pointing out that at present it is very hard to predict any more precise relationship between the radio and X-ray background spectra, because (a) the various classes of source may not make the same relative contributions to the X-ray and radio background, and (b) even a single source may make its main contribution to the two bands at different stages in its evolution. The spectrum of the extragalactic radio background is not in fact known with any precision: one may be mistaken in supposing that it should have the same slope as the mean spectra of resolved sources.

An attractive feature of this model is the way in which it relates the X-ray background to cosmology, to the physics of radio sources, and to radio studies of sources and the background. Painstaking studies of the extragalactic radio background, spectral information on a sample of very faint radio sources, and further understanding of the sources contributing to the counts, should eventually permit us to test the Compton model.

\section{Non-Thermal Bremsstrahlung}

Silk and McCray (1969) have attempted to fit the observed X-ray spectrum by a nonthermal electron bremsstrahlung model in an evolving universe. The process is more efficient at large redshifts because of the higher density of the intergalactic gas. These authors postulate that sub-relativistic electrons with a suitable spectrum are injected at a redshift $\sim 10$. The break in the spectrum results from Coulomb losses, which flatten the electron spectrum at low energies. In order to fit the observed position of

* Note that the energy of the electrons which give rise to X-ray photons of a given energy is independent of the redshift at which the emission occurs, because the cosmological redshift is cancelled by the higher energy of the black body photons which are being scattered.

** In the paper of Felten and Rees (1969), a redshift $\sim 3$ was derived, because the break was presumed to occur at $\sim 60 \mathrm{keV}$ rather than $\sim 20 \mathrm{keV}$. 
the break, they require a cosmology in which the intergalactic gas now has a density of only $\sim 10^{-7} \mathrm{~cm}^{-3}$. They cannot fit the spectrum if the density is higher than this, because losses would then flatten it in the $100 \mathrm{kaV}$ range.

The energy requirements of the postulated burst of electron injection around $z \simeq 10$ are equivalent, in terms of energy input per unit volume, to the production of $6 \times 10^{-2} \mathrm{eV}$ $\mathrm{cm}^{-3}$ at the present epoch. The injected spectrum is $N(E) \propto E^{-2.5}$ particles $\left(\mathrm{cm}^{2} \mathrm{sec}\right.$ ster $\mathrm{keV})^{-1}$. The sources of this enormous flux - for which there is no other evidence are not specified, and the spectrum is chosen in an ad hoc fashion. Also, it is not easy to imagine how the slow electrons could escape from their sources into the low density intergalactic gas before being degraded in a denser medium (in which case the resulting bremsstrahlung spectrum would be altered). If the same sources (radio galaxies or quasars?) also produce relativistic electrons with even $1 \%$ of this energy density, more $\mathrm{X}$-rays would be generated by the Compton process. This is because bremsstrahlung emission is inefficient, whereas Compton scattering may be almost $100 \%$ efficient. A corollary of this low efficiency is that a large amount of heat goes into the thermal gas. It is true that the $\mathrm{X}$-ray emission from a low density intergalactic gas would not be detectable. However the model obviously requires that the thermal particles should be less energetic than the relevant non-thermal ones (i.e. $T \lesssim 10^{7} \mathrm{~K}$ ). If there were 100 times as much energy in protons as in electrons, the gas temperature $T$ would rise to $\sim 10^{9} \mathrm{~K}$ - in this case the distinction between 'thermal' and 'non-thermal' particles would be lost, and there would be no possibility of reproducing the observed $\mathrm{X}$-ray spectrum. Finally, the calculated spectrum, even after all these assumptions have been made, does not seem an adequate fit over the whole range from $1 \mathrm{keV}$.to $1 \mathrm{MeV}$.

Hayakawa (paper in this volume) has developed an analogous model based on bremsstrahlung from protons. As we did not receive any detailed report of this work, we cannot discuss it further. However, because of the similarity to the electron bremsstrahlung process, some of the above remarks apply to this work also. In particular, the problem of the energy requirements seems even more severe.

\section{Soft X-Rays}

There have recently been several observations of diffuse X-rays at energies $\lesssim 1 \mathrm{keV}$. The inconsistencies between the various measurements, which reflect the experimental difficulties, are discussed by Oda (paper in this volume). Soft X-ray observations are especially interesting because of the important role played by galactic (and perhaps also intergalactic) absorption, and also because there may be an additional thermal contribution to the background at these energies.

A straight extrapolation of the power-law spectrum observed at higher energies (1-10 $\AA$ ) yields a flux incident on the galaxy of $\sim 40$ counts at $0.5 \mathrm{keV}$ and $\sim 115$ counts at $0.27 \mathrm{keV}$ (using the spectrum (1')). The flux at the earth attributable to this component would be reduced as a consequence of absorption. The galactic absorption can be estimated either from $21-\mathrm{cm}$ measurements, or directly from the dependence 
of the observed soft X-ray flux on galactic latitude. We return to this problem later.

We may contrast this extrapolation with the flux quoted by Bunner et al. (paper in this volume) which, after their correction for galactic absorption has been applied, is $\sim 50$ counts at $0.5 \mathrm{keV}$ and $\sim 120$ counts at $0.27 \mathrm{keV}$. The corrected flux at $0.28 \mathrm{keV}$ quoted by Bowyer et al. (1968) is $\sim 200$ counts.* Although other observers (Henry et al., 1968; Baxter et al., 1969) have claimed that the flux incident on the Galaxy is somewhat higher, we do not consider that the observations have yet established the definite existence of any component in addition to the non-thermal background observed at higher energies.

If there were such a component, however, it could be interpreted as bremsstrahlung emission from an ionised gas at a temperature $\sim 10^{6} \mathrm{~K}$. Indeed Henry et al. (1968), who quoted a corrected flux of 600 counts at $0.28 \mathrm{keV}$ - too high to be a plausible extrapolation of the non-thermal spectrum - attributed the excess to an intergalactic medium with a density $\sim 10^{-5} \mathrm{~cm}^{-3}$. If correct, this would be the first positive evidence for the existence of a quantity of material sufficient to close the universe, and would plainly be exceedingly important for cosmology. Weymann (1967) has considered in detail the 'thermal history' of an intergalactic gas, assuming it to consist of hydrogen and helium (in the ration $10: 1$ by number). Present observations set two important constraints on the state of such a gas: it must be very highly ionized so as to account for the lack of absorption shortward of the redshifted Lyman $\alpha$ wavelength in the spectra of distant quasars (Gunn and Peterson, 1965), and so (at least if it is collisionally ionised) it must be hot; on the other hand, it must not be so hot that it emits thermal X-rays with an intensity exceeding the observed background at any wavelength. Weymann showed that if the present density of this gas were $\sim 10^{-5} \mathrm{~cm}^{-3}$, the 'thermal history' was constrained within very narrow limits by the data available at that time (1967). Although one of Weymann's models did indeed predict a soft $\mathrm{X}$-ray flux comparable with that reported by Henry et al., we wish to emphasise that this is only one of several possible origins for thermal bremsstrahlung of this intensity. (A fuller discussion of this and related topics is given by Rees et al., 1968). All that is required is a gas with emission measure $\sim 0.3 \mathrm{~cm}^{-6} \mathrm{pc}$ in a typical direction, at a temperature $\sim 5 \times 10^{5} \mathrm{~K}$ (or $\sim 5 \times 10^{5}(1+z) \mathrm{K}$ if the main emission comes from a substantial redshift $z$ ). This emission measure could be furnished by gas of density $\sim 10^{-4} \mathrm{~cm}^{-3}$ within clusters of galaxies. Another possibility would be emission from the halo of our Galaxy (with density $\sim 10^{-2} \mathrm{~cm}^{-3}$ and dimensions $\sim 10^{4} \mathrm{pc}$ ), or possibly smaller and denser regions of gas with $T \sim$ a few times $10^{6} \mathrm{~K}$, such as might be expected behind shock fronts associated with the high velocity clouds (Savedoff et al., 1967). In the latter cases the required emission measure could be even lower than $0.3 \mathrm{~cm}^{-6} \mathrm{pc}$, because we would expect heavy elements also to be present, and these emit more efficiently than hydrogen and helium when $T \simeq 10^{6} \mathrm{~K}$ (Pottasch, 1966).

Shklovskii (1969) has proposed that it could be due to emission lines of O vII and OvIII (with $\lambda \simeq 20 \AA$ ) from supernovae in galaxies with $z \simeq 1$. Finally, the excess soft

prAn additional uncertainty in these quoted fluxes arises because the figure itself depends on the

* esumed spectrum over the broad energy band covered by the detector. 
X-ray flux could be the integrated effect of a population of extragalactic sources which mimic a thermal spectrum.

An indirect method of estimating how the soft X-ray spectrum extends into the ultraviolet has been proposed by Sunyaev (1969). He argues that the low density HI which has been detected in the outlying parts of M 31 and other nearby galaxies would have been dispersed by an inward-propagating ionisation front if the extragalactic ultraviolet radiation were too intense. Sunyaev claims that the bremsstrahlung curve derived by Weymann (1967), which was consistent with the $0.28 \mathrm{keV}$ flux measured by Henry et al., can be ruled out by this argument. However, Bunner et al., whose observations in two energy bands gave them some spectral information, found that any thermal component of the background would not have such a steep slope as the spectrum given by Weymann's models, and, when extrapolated down to the Lyman limit, is actually compatible with the upper limit given by Sunyaev.

Also, it may be possible to evade Sunyaev's conclusions by supposing that the observed neutral hydrogen is surrounded by an ionised region (perhaps filling the volume of the Local Group) which shields the $\mathrm{H}_{\mathrm{i}}$ from the external ultraviolet flux. We have considered this point further in another paper in this volume.

\section{A. GALACTIC ABSORPTION}

A straightforward estimate of the X-ray opacity of the Galaxy can be made by using $21-\mathrm{cm}$ observations of the column density of neutral hydrogen as a measure of the amount of matter along the line of sight. The most accurate calculations of the absorption cross-sections have been carried out by Bell and Kingston (1967). At energies below the $\mathrm{K}$-edge of carbon $(0.28 \mathrm{eV})$ the absorption is mainly due to hydrogen and helium. Following Bowyer et al. (1968), we may write the absorption cross-section per hydrogen atom in the form

where

$$
\sigma(E)=\sigma_{0}\left(\begin{array}{c}
E \\
E_{0}
\end{array}\right)^{-3.2} \mathrm{~cm}^{2}
$$

$$
\sigma_{0}=(0.84+21.4 \xi) \times 10^{-21} \mathrm{~cm}^{2}
$$

$\sigma_{0}$ is the effective cross-section at $0.28 \mathrm{keV}$, and $\xi=n(\mathrm{He}) / n(\mathrm{H})$. If we assume that $\xi$ has the 'cosmical' value of $0.1, \sim 70 \%$ of the absorption would be due to helium. (There may be some extra opacity from molecular hydrogen.) According to Bowyer et al. and Bunner et al., the background at $0.28 \mathrm{keV}$ decreases more slowly with angular distance from the galactic pole than would be expected on the basis of the $\mathrm{HI}_{\mathrm{I}}$ column density derived from $21-\mathrm{cm}$ data. Unless interstellar space is almost devoid of helium, one must assume that either (a) the straightforwardly calculated galactic opacity is misleadingly high, or (b) local sources are responsible for some of the background at low galactic latitudes.

(a) (i) 21-cm maser action.

In an attempt to account for the weak Lyman $\alpha$ absorption in some stellar spectra (Morton, 1967; Jenkins and Morton, 1967; Carruthers, 1968; Morton and Jenkins, 
1968) Fischel and Stecher (1967) suggested that 'pumping' by blue-shifted Lyman $\alpha$ photons could lead to population inversion in the hyperfine states of HI. Because $21-\mathrm{cm}$ radiation would be enhanced by the ensuing maser action, a lower column density of $\mathrm{H} \mathrm{I}$ is then sufficient to produce a given intensity in the $21-\mathrm{cm}$ line. (A similar suggestion had earlier been made by Shklovskii (1967), following Varshalovich (1967), for the specific case of the high-velocity clouds.) A more detailed study (Van Bueren and Oort, 1968; Storer and Sciama, 1968), however, shows that, because of the short mean free path of Lyman $\alpha$ photons in Hi regions, this effect only occurs in thin layers, and so could not significantly affect the $21-\mathrm{cm}$ intensity along a whole line of sight through the galactic disc. This proposed explanation for the anomalous latitudedependence of the X-ray background must consequently be discarded.

(ii) 'Clumpiness' of interstellar hydrogen.

High resolution $21-\mathrm{cm}$ studies at low galactic latitudes reveal irregularities which suggest that interstellar hydrogen may be in clouds, the precise nature of which is, however, still unclear (Van Woerden, 1967). A given amount of hydrogen would obviously tend to absorb X-rays less if it were concentrated into clouds. This is because part of the hydrogen which contributes to the $21-\mathrm{cm}$ emission would be embedded in clouds which were already so opaque that its additional X-ray absorbing power is not used effectively. For this to be an important effect, a substantial fraction $(\gtrsim 60 \%)$ of the HI must be in large clouds. Bowyer and Field (1969) considered clouds with column densities up to $(1-5) \times 10^{21} \mathrm{~cm}^{-2}$, and showed that the absorption, averaged over a solid angle large enough to contain a statistical sample of clouds, would be only $0.33 \pm 0.18$ of the value if the same amount of hydrogen were smoothly distributed. This reduction would be sufficient to account for the latitude-dependence which Bowyer et al. observed. The beam used by these workers was $8^{\circ} \times 8^{\circ}$. However, the large clouds discussed by Bowyer and Field would be $\sim 25$ pc across (Van Woerden, 1967), so that at distances $100-300$ pc they would subtend angles larger than the beam. This fact seems to us to raise questions regarding the validity of Bowyer and Field's discussion. Bunner et al. included the effects of 'clumpiness' in their analysis, and considered clouds with column density $1.2 \times 10^{21} \mathrm{~cm}^{-2}$, which would be opaque at $0.28 \mathrm{keV}$ but not at $0.5 \mathrm{keV}$. They used a beam $2.6^{\circ} \times 23^{\circ}$ at half power, but it is not clear to us, from the paper of Bunner et al., whether their work is subject to the above objection.

As is remarked by Bunner et al., the large hydrogen clouds may be opaque in the $21-\mathrm{cm}$ line, particularly if the spin temperature is low. Should this be so, their column density may have been underestimated. If this effect were properly allowed for, it is by no means obvious whether the assumption that the observed $\mathrm{HI}$ is in clouds actually reduces the calculated X-ray opacity at all.

(b) Any galactic contribution to the soft X-ray flux must come effectively from distances $\lesssim 200 \mathrm{pc}$, because most $0.28 \mathrm{keV}$ photons emitted at greater distances would be absorbed before reaching us. The existence of a galactic component could account for the observed latitude-dependence of the diffuse soft X-rays without the necessity to invoke anomalous absorption. If such a background were the integrated effect of 
unresolved sources, each source must be much fainter than the known discrete galactic sources. For none to have been resolved by Bunner et al., the density of sources would need to be $\gtrsim 10^{-2} \mathrm{pc}^{-3}$.

Whether such a component is due to sources or is genuinely diffuse, its spectrum must be steeper than that of the extragalactic background. Otherwise the anisotropy above $2 \mathrm{keV}$ (at which energies the whole disc is transparent) would be much greater than that reported by Cooke et al. (paper in this volume). The latitude-dependence of the expected galactic flux depends on whether the scale height of the emission is larger or smaller than the scale height of the interstellar gas responsible for the absorption (Rees, paper in this volume).

\section{B. INTERGALACTIC ABSORPTION}

All the theories for the X-ray background which we have discussed imply that the bulk of the observed radiation originates at cosmological distances $(z \simeq 1)$ : in models involving evolution, the observed photons may even have originated at redshifts $z \gtrsim 5$. If a substantial part of the $\mathrm{X}$-ray background at $0.28 \mathrm{keV}$ is an extension of the nonthermal background at high energies, we may infer upper limits to the amount of intergalactic absorption. The condition that the optical depth out to $z \simeq 1$ should be $\lesssim 1$ requires that the mean density of neutral hydrogen must be $\lesssim 10^{-7} \mathrm{~cm}^{-3}$, so that, if the intergalactic gas has the 'magic' density of $\sim 10^{-5} \mathrm{~cm}^{-3}$ it must be $\gtrsim 99 \%$ ionised.* Although the Lyman $\alpha$ observations of quasars set a much more stringent limit, this is a non-trivial additional constraint, because (a) it is independent of the cosmological interpretation of quasar redshifts, and (b) some theoretical 'thermal histories' would allow the gas to partially recombine by the present time, even if the ionisation at $z \simeq 2$ were essentially complete.

If the X-rays originate at redshifts much greater than 2 , we can infer something about the ionisation level of the gas beyond the remotest observed quasars. (In calculating the absorption at very large redshifts, we must allow for the facts that the gas density $\propto(1+z)^{3}$, that the cross-section for absorption of a photon of observed wavelength $\lambda$ by hydrogen or helium is roughly proportional to $\lambda^{3}$ (i.e. to $(1+z)^{-3}$ ), and that the effective path length is $(1+z)^{-3 / 2}$ in an Einstein-de Sitter model and $(1+z)^{-1}$ in a low-density model.) The combined result of these effects is that the limits we can place on the degree of ionisation are somewhat less sensitive than for smaller redshifts. These conclusions would not alter significantly if the gas were concentrated in clusters, because there would be several clusters along every line of sight out to a redshift $z \simeq 2$.** If an accurate spectrum of the X-ray background from, say, 1-50 $\AA$ were available, one might be able to infer whether the long wavelength flux was being attenuated and, if so, where the absorption was taking place.

Limits can also be placed on the intergalactic abundance of heavy elements. At

* Some possible effects of scattering by ionised intergalactic gas, which may be important if the X-rays originate at $z \gtrsim 5$, are discussed by us elsewhere (paper by Rees and Setti, in this volume).

** Specially large clusters may be detectable in absorption at $1-3 \mathrm{keV}$ if they contain a large amount of gas. 
temperatures $\lesssim 10^{6} \mathrm{~K}$, the $\mathrm{K}$-electrons of elements such as $\mathrm{CNO}$ would not be stripped off by collisions with electrons. If the optical depth out to $z \simeq 1$ is $\lesssim 1$ at $0.28 \mathrm{keV}$, these elements could not have more than $\sim 2 \%$ of their 'cosmical' abundance if the total density is $\sim 10^{-5} \mathrm{~cm}^{-3}$ (with correspondingly less sensitive limits for lower densities). While it would be surprising if these elements were present with their full local abundance relative to hydrogen, this is nevertheless a significant result, since it limits the cumulative contamination of intergalactic space by galaxies and exploding objects during the expansion of the universe.*

\section{6. $\gamma$-Rays}

Various physical processes which might give rise to cosmic $\gamma$-ray emission have been described in various papers (Ginzburg and Syrovatskii, 1964a, b; Gould and Burbidge, 1965; Hayakawa et al., 1964; Oda, 1965; Fazio, 1967; Garmire and Kraushaar, 1965). The present data indicate an isotropic flux at $\sim 100 \mathrm{MeV}$ (Clark et al., 1968) and also a flux at 1-10 MeV observed with a non-directional detector (Vette et al., paper in this volume). There are also upper limits to the background at much higher energies (Oda, 1965). The $100 \mathrm{MeV}$ flux lies on an extrapolation of the X-ray spectrum $\left(1^{\prime \prime}\right)$, and is possibly due to the same process. Although the Compton spectrum must cut off at some energy, there seems no reason why it should not extend up to $100 \mathrm{MeV}$. Electrons with $\gamma=3 \times 10^{5}$ would be required to produce these photons via Compton scattering of black body photons.

From time to time, various other processes have been proposed for producing $\gamma$-rays. Among these we may mention decay of $\pi_{0}$ produced in collisions of high-energy cosmic rays with intergalactic matter. Even if the gas density is $10^{-5} \mathrm{~cm}^{-3}$, a rather high intergalactic flux of cosmic rays would be needed to produce the observed $\gamma$-rays (Gould, 1967). Another possibility is matter-antimatter annihilation (Stecker, 1969). It would be premature to discuss these processes in detail until more data are available.

\section{Conclusions}

Our primary conclusion is that there is not yet any reliably established theory for the $\mathrm{X}$-ray background. It should be superfluous to emphasise the necessity for further

* In a recent preprint, Longair and Sunyaev (1969) have proposed a unified model to account for the spectrum of the whole X-ray background. They suppose that relativistic electrons are generated in a class of sources, which they tentatively identify with Seifert galaxies. It is envisaged that the electrons are confined in the nucleus for a period during which losses cause a break to develop in their spectrum. During this stage they emit X-rays by Compton scattering of infrared radiation, and it is suggested that the X-ray background above $\sim 1 \mathrm{keV}$ is the integrated effect of such emission in all sources of this class. The electrons then escape, and emit soft X-rays by Compton scattering of the $3 \mathrm{~K}$ background. In order to obtain the observed energy in the soft X-ray background, it must be assumed that the electrons avoid serious adiabatic losses during their escape. Also, the model requires remarkably 'standardised' conditions in all the sources, otherwise the bends in the $\mathrm{X}$-ray background spectrum would have been smeared out. Finally if these sources were indeed Seyferts, and since $1-2 \%$ of all the galaxies are of the Seyfert type, NGC 1068, e.g., would be likely to appear at least as strong as M 87 at X-ray energies. 
observations before a firmer understanding can be achieved. At present it is impossible to discriminate decisively between alternative models, any of which can be made compatible with the present data. We have concentrated our discussion on the astrophysical implications of the various possibilities, and on the plausibility of the assumptions made in each of them. At the moment it seems that the model which entails the minimum number of ad hoc assumption is Compton scattering of black body photons by relativistic electrons in radio galaxies at large redshifts. We are doubtless guilty of oversimplification in seeking an explanation in terms of any single mechanism: indeed, we cannot discount the possibility that all the processes discussed here may contribute significantly to the $\mathrm{X}$-ray background.

There is need for an improved spectrum in the $1 \mathrm{keV}-1 \mathrm{MeV}$ range, in order to pin down the position of the apparent 'break'. More observations are required in order to clarify the confusing situation below $1 \mathrm{keV}$, and, at the other extreme, information in the $\gamma$-ray region is still lacking. High-resolution studies below $1 \mathrm{keV}$, in conjunction with improved $21-\mathrm{cm}$ maps at high galactic latitudes, should indicate whether the galactic absorption is due to small and dense clouds. It should then be possible to estimate what contribution to the flux at low galactic latitudes comes from local sources, or from diffuse processes occurring within the disc.

There is also a need for high-resolution observations, and studies of the level of isotropy on various angular scales.

Apart from M 87, and perhaps the Large Magellanic Cloud (Mark et al., 1969) and 3C 273 (Friedman and Byram, 1967) there are no data on the X-ray emission from any discrete objects beyond our own Galaxy. Until this state of affairs has changed, it is difficult to estimate the likely contribution of such sources to the background.

Attempts to detect X-rays from rich clusters (e.g., Virgo or Coma) would provide a better estimate of the mean output of normal galaxies. (Low energy observations could also yield information about gas within the clusters.) If sources do indeed contribute appreciably to the X-ray background, observations with good angular resolution may tell us the number and power of the objects involved. (Some relevant figures are quoted by Weymann, 1968). Some 'patchiness' would also be expected in the so-called 'diffuse' processes, because neither the fast particles (nor, in the case of bremsstrahlung, the thermal gas) is likely to be distributed smoothly enough for the emission to appear completely uniform. In particular, Compton scattering of the black-body background, normally thought of as a diffuse process, would in fact give rise to apparently discrete sources, simply because at large redshifts the Compton lifetimes may be only $\sim 10^{6}$ years, and the electrons do not have time to escape far from their point of origin. It may be possible to resolve such sources with $z \simeq 2-5$ (Felten and Rees, 1969).

\section{Acknowledgements}

We have benefited from discussions with many colleagues, and would especially like to thank Drs. J. E. Felten and D. W. Sciama. One of us (G.S.) acknowledges support from CNR. 


\section{References}

Allen, C. W.: 1963, Astrophysical Quantities, The Athlone Press, University of London.

Baxter, A. J., Wilson, B. G., and Green, D. W.: 1969, Astrophys. J. 155, L145 (see also paper in this Symposium).

Bell, K. L. and Kingston, A. E.: 1967, Monthly Notices Roy. Astron. Soc. 130, 373.

Bergamini, R., Londrillo, P., and Setti, G.: 1967, Nuovo Cimento 52B, 495.

Boldt, E. and Serlemitsos, P.: 1969, Astrophys. J. 157, 557.

Bowyer, C. S., Field, G. B., and Mack, J. E.: 1968, Nature 217, 32.

Bowyer, C. S. and Field, G. B.: 1969, Nature 223, 573.

Brecher, K. and Morrison, P., 1967: Astrophys. J. 150, L61.

Brecher, K. and Morrison, P.: 1969, Phys. Rev. Letters 23, 802.

Bridle, A. H.: 1967a, Monthly Notices Roy. Astron. Soc. 136, 219.

Bridle, A. H.: 1967b, Observatory 87, 263.

Carruthers, G. R.: 1968, Astrophys. J. 151, 269.

Clark, G. W., Garmire, G. P., and Kraushaar, W. L.: 1968, Astrophys. J. 153, L203.

Cooke, B. A., Griffiths, R. E., and Pounds, K. A.: 1969, Nature 224, 134. (See also paper in this volume.)

Davidson, W. and Davies, M.: 1964a, Monthly Notices Roy. Astron. Soc. 127, 241.

Davidson, W. and Davies, M.: 1964b, Monthly Notices Roy. Astron. Soc. 128, 363.

Fazio, G. G.: 1967, Ann. Rev. Astron. Astrophys. 5, 481.

Felten, J. E. and Morrison, P.: 1966, Astrophys. J. 146, 686.

Felten, J. E. and Rees, M. J.: 1969, Nature 221, 924.

Fischel, D. and Stecher, T. P.: 1967, Astrophys. J. 150, L51.

Friedman, H. and Byram, E. T.: 1967, Science 158, 257.

Garmire, G. P. and Kraushaar, W. L.: 1965, Space Sci. Rev. 4, 123.

Ginzburg, V. L. and Syrovatskii, S. I.: 1964a, Usp. Fiz. Nauk. SSSR 84, 201 [transl. Soviet Phys. Usp. 7, 1965, 696].

Ginzburg, V. L. and Syrovatskii, S. I.: 1964b, Origin of Cosmic Rays, Pergamon Press, Oxford.

Gorenstein, P., Kellog, E. M., and Gursky, H.: 1969, Astrophys. J. 156, 315.

Gould, R. J. and Burbidge, G. R.: 1965, Ann. Astrophys. 28, 171.

Gould, R. J.: 1967, Amer. J. Phys. 35, 376.

Gunn. J. E. and Peterson, B. A.: 1965, Astrophys. J. 142, 1633.

Hayakawa, S. and Matsuoka, M.: 1964, Progr. Theoret. Phys. (Kyoto), Suppl. 30, 204.

Hayakawa, S., Okuda, H., Tanaka, Y., and Yamamoto, Y.: 1964, Progr. Theoret. Physics, Suppl. $30,153$.

Haymes, R. C., Ellis, D. V., Fishman, G. J., Glenn, S. W., and Kurfess, J. D.: 1969, Astrophys. J. 155, L31.

Henry, R. C., Fritz, G., Meekins, J. F., Friedman, H., and Byram, E. T., 1968, Astrophys. J. 153, L11.

Hornby, J. M. and Williams, P. J. S.: 1966, Monthly Notices Roy. Astron. Soc. 131, 237.

Jenkins, E. B. and Morton, D. C.: 1967, Nature 215, 1257.

Kellermann, K. I.: 1966, Astrophys. J. 146, 621.

Kellermann, K. I. and Pauliny-Toth, I. I. K.: 1969, Astrophys. J. 155, L71.

Lang, K. R. and Terzian, Y.: 1969, Astrophys. Letters 3, 29.

Longair, M. S.: 1966, Monthly Notices Roy. Astron. Soc. 133, 421.

Longair, M. S. and Sunyaev, R. A.: 1969, preprint No. 84, Lebedev Physical Institute, Moscow.

Low, F. J. and Tucker, W. H.: 1968, Phys. Rev. Lett. 21, 1538.

Mark, H., Price, R., Rodrigues, R., Seward, F. D., and Swift, C. D.: 1969, Astrophys. J. 155, L143.

Morton, D. C.: 1967, Astrophys. J. 147, 1017.

Morton, D. C. and Jenkins, E. B.: 1968, Astron. J. 73, S110.

Oda, M.: 1965, Proc. Int. Conf. Cosmic Rays, London, Vol. I, p. 68.

Oort, J. H.: 1958, 'La structure et l'évolution de l'Univers', Brussels, Solvay Conference.

Pooley, G. G. and Ryle, M.: 1968, Monthly Notices Roy. Astron. Soc. 139, 515.

Pottasch, S. R.: 1966, Bull. Astron. Inst. Netherl. 18, 156.

Rees, M. J.: 1967, Monthly Notices Roy. Astron. Soc. 137, 429.

Rees, M. J., Sciama, D. W., and Setti, G.: 1968, Nature 217, 326.

Rees, M. J. and Setti, G.: 1968, Nature 219, 127. 
Rowan-Robinson, M.: 1967, Nature 216, 1289.

Rowan-Robinson, M.: 1968, Monthly Notices Roy. Astron. Soc. 138, 445.

Ryle, M.: 1968, Ann. Rev. Astron. Astrophys. 6, 249.

Savedoff, M. P., Hovenier, J. W., and Van Leer, B.: 19̀67, Bull. Astron. Inst. Netherl. 19, 107.

Sciama, D. W.: 1968, Review paper, presented at the X-Ray Meeting of the Royal Society, London, November 1968. To appear in Phil. Trans. Roy. Soc.

Schmidt, M.: 1966, Astrophys. J. 146, 7.

Schmidt, M.: 1968, Astrophys. J. 151, 393.

Seward, F. D., Chodil, G., Mark, H., Swift, C. D., and Toor, A.: 1967, Astrophys. J. 150, 845.

Shapiro, M. H. and Silberberg, R.: 1968, Proc. 10th Internat. Conf. Cosmic Rays, Calgary, Canadian Jour. Phys. 46, S561.

Shklovskii, I. S.: 1967, Soviet Astron. - A.J. 11, 240.

Shklovskii, I. S.: 1969, Astrophys. Letters 3, 1.

Sholomitskii, G. B.: 1968, Soviet Astron. - AJ 11, 756.

Silk, J.: 1968, Astrophys. J. 151, L19.

Silk, J.: 1969, Nature 221, 347.

Silk, J. and Mc Cray, R.: 1969, Astrophys. Letters 3, 59.

Stecker, F. W.: 1969, Nature 221, 425.

Storer, S. H. and Sciama, D. W.: 1968, Nature 217, 1237.

Sunyaev, R. A.: 1969, Astrophys. Letters 3, 33.

Turtle, A. J. and Baldwin, J. E.: 1962, Monthly Notices Roy. Astron. Soc. 124, 459.

Varshalovich, D. A.: 1967, Soviet Phys. - JETP 25, 157.

Van Bueren, H. G. and Oort, J. H.: 1968, Bull. Astron. Inst. Netherl. 19, 414.

Van Woerden, H.: 1967, in Radio Astronomy and the Galactic System, I.A.U. Symp. No. 31 (ed. by H. Van Woerden), p. 3.

Véron, P.: 1966a, Nature 211, 724.

Véron, P.: 1966b, Ann. Astrophys. 29, 231.

Weymann, R. J.: 1967, Astrophys. J. 147, 887.

Weymann, R. J.: 1968, in Highlights of Astronomy, I.A.U. Publication (ed. by L. Perek), Reidel Publ. Comp., Dordrecht, Holland, p. 220.

Williams, P. J. S.: 1966, Nature 210, 285. 